\title{
Retorno al deporte luego de una cirugía de Latarjet: revisión sistemática de la bibliografía
}

\author{
Ignacio Tanoira, Luciano Rossi, Juan Víctor A. Franco, Camila M. Escobar Liquitay, Ignacio Pasqualini, \\ Maximiliano Ranalletta \\ Unidad de Patología de Hombro, Servicio de Ortopedia y Traumatología, Instituto de Ortopedia y Traumatología \\ "Prof. Dr. Carlos E. Ottolenghi”, Hospital Italiano de Buenos Aires, Ciudad Autónoma de Buenos Aires, Argentina
}

\begin{abstract}
RESUMEN
Introducción: El objetivo de este estudio fue describir las tasas de retorno al deporte y el nivel alcanzado por los pacientes con inestabilidad de hombro luego del procedimiento abierto y artroscópico de Latarjet. Materiales y Métodos: Seguimos un protocolo prespecificado y registrado en PROSPERO. Evaluamos la calidad de los estudios y utilizamos el sistema GRADE para evaluar la calidad general de la evidencia obtenida en los resultados. Incluimos estudios que evalúan el retorno al deporte de los pacientes luego de una cirugía de Latarjet con un seguimiento mínimo de 2 años. Resultados: Se incluyeron 24 estudios, con 1436 atletas, todos con cirugía y un seguimiento promedio de 57 meses (rango 24-240). La tasa general de retorno al deporte varió del $65 \%$ al $100 \%$ de los pacientes, de ellos, el $23-100 \%$ retornó al mismo nivel. El tiempo promedio de retorno al deporte fue de 6 meses (rango 1-36). El nivel de evidencia fue bajo debido a las características de los estudios incluidos (nivel de evidencia IV), las limitaciones de los estudios y sus inconsistencias. Conclusiones: La mayoría de los atletas con luxación recidivante de hombro sometidos a una cirugía de Latarjet retoman la práctica deportiva; sin embargo, el nivel alcanzado varía sustancialmente. El tiempo promedio de retorno al deporte fue de 6 meses, y no hubo diferencias significativas entre los deportistas competitivos y recreacionales.
\end{abstract}

Palabras clave: Inestabilidad glenohumeral; retorno al deporte; Latarjet.

Nivel de Evidencia: IV

Return to Sports after Latarjet Surgery: Systematic Literature Review

\section{ABSTRACT}

Background: The purpose of this study was to describe rates of return to sports and the level achieved by patients after a Latarjet procedure. Methods: We followed a protocol registered in PROSPERO (registration number CRD42018107606). A literature search was performed in May 2019 in MEDLINE, EMBASE, CENTRAL and clinical trials records. We used the GRADE approach for the assessment of the overall quality of the evidence per outcome. We included studies (evidence level I to IV) evaluating return to sports following shoulder stabilization with the Latarjet procedure with a minimum of 2-year follow-up. Results: We included 24 studies, including 1436 athletes, all treated surgically after an average follow-up of 57 months (range 24 to 240). The overall rate of return to sport ranged from $65 \%$ to $100 \%$, including $23 \%$ to $100 \%$ at an equivalent level of play. The average time for return to sport was 6 months (range, 1 - 36 months). Competitive athletes appeared to return to the same level of competition and this difference was not statistically significant $(p=0.32)$. The quality of the evidence was very low due to study design (evidence level IV), study limitations and inconsistency. Conclusion: Most athletes with glenohumeral instability returned to sport, however the level maintained after shoulder stabilization with the Latarjet procedure varied substantially. The average time to return to sports was 6 months and results were equally favorable in competitive and recreational athletes.

Key words: Glenohumeral instability; return to sports; Latarjet.

Level of Evidence: IV

Recibido el 4-3-2020. Aceptado luego de la evaluación el 28-9-2020 • Dr. IGNACIO TANOIRA • ignacio.tanoira@ hiba.org.ar

https://orcid.org/0000-0002-2869-2390

Cómo citar este artículo: Tanoira I, Rossi L, Franco JVA, Escobar Liquitay CM, Pasqualini I, Ranalletta M. Retorno al deporte luego de una cirugía de Latarjet: revisión sistemática de

la bibliografía. Rev Asoc Argent Ortop Traumatol 2021;86(2):263-280. https://doi.org/10.15417/issn. 1852-7434.2021.86.2.1068 


\section{INTRODUCCIÓN}

El mejor manejo de la inestabilidad recidivante de hombro con un defecto óseo glenoideo significativo sigue siendo un desafío. ${ }^{1}$ Se observan defectos óseos glenoideos y humerales en más del $90 \%$ de las luxaciones recidivantes de hombro. ${ }^{2}$ Las altas tasas de recurrencia (30-60\%) luego de la reparación artroscópica o abierta de Bankart en pacientes con defectos óseos significativos en la glena, el húmero o combinados, llevan a muchos cirujanos a elegir procedimientos de reconstrucción ósea glenoidea para el manejo de esta enfermedad. ${ }^{3-5}$ Entre estas, la técnica utilizada más popular es la cirugía de Latarjet. ${ }^{5,6}$ En este procedimiento, tres efectos trabajan en conjunto con el objetivo de mejorar la estabilidad articular, logrando un "triple efecto" de estabilización. ${ }^{1}$ Primero, el hueso coracoideo aumenta la superficie glenoidea funcionando como restrictor estático, mejorando el arco seguro necesario para la translación humeral antes de la luxación (efecto óseo). En segundo lugar, el tendón conjunto actúa realizando un efecto de cincha sobre la cabeza humeral, limitando la translación anterior cuando el hombro está en abducción y en rotación externa (efecto cincha). En tercer lugar, el labrum y la cápsula anterior son reinsertados en la glena y reforzados mediante el ligamento coracoacromial (efecto paragolpe). ${ }^{7.8}$

Si bien el procedimiento de Latarjet logra probados resultados para el manejo de la inestabilidad recidivante en la población general, ${ }^{2,6,9-11}$ existe poca información en relación con el retorno al deporte y el nivel de retorno. Unas de las principales expectativas de los deportistas, al margen de la edad y el nivel de competición, es volver a practicar el deporte lo antes posible y al mismo nivel que antes de la lesión. La técnica quirúrgica elegida debe lograr no solo un hombro estable, sino también un retorno al deporte seguro. En consecuencia, este subgrupo de pacientes representa un desafío para el cirujano de hombro. ${ }^{12}$

No hemos encontrado revisiones bibliográficas sistemáticas que evalúen específicamente el retorno al deporte luego de una cirugía de Latarjet abierta o artroscópica. Como las series publicadas sobre esta técnica son pequeñas, realizar este tipo de estudios permite brindar información sólida a cirujanos y pacientes. El objetivo de este estudio fue efectuar una revisión sistemática de la bibliografía para describir las tasas de retorno al deporte y el nivel alcanzado por los pacientes con inestabilidad de hombro luego del procedimiento de Latarjet.

\section{MATERIALES Y MÉTODOS}

Este estudio se llevó a cabo de acuerdo con las Expectativas Metodológicas de las Revisiones Sistemáticas de Intervenciones (Methodological Expectations of Systematic Reviews of Interventions) ${ }^{13}$ y el Manual Cochrane. ${ }^{14} \mathrm{El}$ informe sigue la declaración PRISMA (Preferred Reporting Items for Systematic reviews and Meta-Analyses). ${ }^{15}$ El protocolo de esta revisión se registró en la base de datos de registro de revisiones sistemáticas, PROSPERO (número de registro CRD42018107606).

\section{Estrategia de búsqueda}

El 24 de mayo de 2019 se realizó una búsqueda bibliográfica electrónica en las bases de datos MEDLINE a través de PubMed, Embase a través de Elsevier y CENTRAL a través de la Biblioteca Cochrane, la Plataforma Internacional de Registro de Ensayos Clínicos (ICTRP) y clinictrials.gov. Además, se verificaron las referencias para cada artículo, y se realizó una búsqueda manual de artículos que posiblemente podrían incluirse en el análisis.

\section{Criterios de selección}

Los criterios de inclusión fueron: estudios que, al margen del idioma o los niveles de evidencia, ${ }^{1-4}$ evaluaron el retorno al deporte y los resultados clínicos después del tratamiento de una inestabilidad anterior recurrente del hombro y un defecto óseo glenoideo en atletas adultos, mediante un procedimiento abierto o artroscópico de Latarjet. Los resultados primarios incluyeron: 1) retorno al deporte, definido como el tiempo para regresar a las actividades deportivas, 2) retorno al mismo nivel de competición, definido como la tasa de pacientes que regresan al mismo nivel de competencia que tenían antes de la lesión. Los resultados adicionales incluyeron: el tiempo para regresar a los deportes, la tasa total de complicaciones y la tasa total de pacientes sometidos a procedimientos adicionales (revisiones).

Los criterios de exclusión fueron: revisiones bibliográficas, opiniones de expertos, estudios no clínicos, reportes de casos y estudios clínicos que no evaluaron a deportistas o el regreso al juego. Asimismo, se excluyeron los estudios que incluían pacientes con otros tipos de inestabilidad (p. ej., posterior o voluntaria) o sin evaluaciones clínicas o radiográficas en un seguimiento final mínimo de dos años. Dos autores (IT y LR) seleccionaron los resúmenes y, luego, los analizaron por separado. Cuando el resumen era pertinente, se analizó el artículo completo. En caso de desacuerdo entre los autores, se buscó un consenso. Si no se llegó a un consenso, se consultó a un tercer autor (MR). 


\section{Evaluación de la calidad de los estudios}

La calidad de los estudios incluidos se evaluó de acuerdo con la herramienta de evaluación de calidad para series de casos desarrollada por el Instituto de Economía de la Salud. ${ }^{16}$ Esta herramienta fue desarrollada y validada específicamente para evaluar la calidad de las series de casos. Incluye 18 ítems que cubren el objetivo del estudio, las características de la población, la intervención, la medición de resultados, el análisis estadístico, los resultados y las conclusiones, los conflictos de intereses y las fuentes de apoyo. Esta evaluación fue realizada, de forma independiente, por dos autores, llegando a un consenso en caso de desacuerdo. Integramos estas evaluaciones de la calidad de la realización de los estudios para resumir la calidad general de la evidencia considerando la consistencia de los hallazgos, la exactitud de la evidencia, la precisión de los resultados y la evidencia del sesgo de publicación de acuerdo con la guía proporcionada por el Manual GRADE que presenta una guía para evaluar la calidad de la evidencia y así graduar la fuerza de los resultados. ${ }^{17}$ Resumimos estas evaluaciones en las tablas de resumen de resultados utilizando el programa GRADEpro. ${ }^{18}$

\section{Extracción de datos y síntesis}

La extracción de datos se basó en una forma estandarizada que fue predefinida de acuerdo con el protocolo. Dos autores (IT y LR) extrajeron los datos de forma independiente. En caso de desacuerdo entre ellos, se buscó un consenso. Si no se llegó a un consenso, se consultó a un tercer autor (MR). Incluyó 1) las características del estudio (diseño, año y cantidad de pacientes), 2) las características de los participantes (edad, sexo, deporte practicado, nivel de deportes), 3) las características de la inestabilidad del hombro (gravedad y tamaño del defecto óseo, retraso entre la lesión y la cirugía), 4) los resultados clínicos del seguimiento final, 5) rehabilitación y criterios para el regreso al deporte y 6) datos de resultados primarios y secundarios.

Los datos fueron analizados con el programa STATA. Se pretendió utilizar un modelo de efectos aleatorios para agrupar proporciones. ${ }^{19} \mathrm{El}$ porcentaje de variación total entre los estudios debido a la heterogeneidad se evaluó mediante la medida I. ${ }^{20}$ Los umbrales utilizados fueron "bajo" (0-40\%), "moderado" (30-60\%), "sustancial" (50$90 \%$ ) y considerable (75-100\%). La tasa media de retorno al deporte se calculó con un intervalo de confianza del $95 \% .{ }^{21}$ Las tasas de retorno al deporte en los diferentes estudios, así como la tasa combinada, se representaron en un diagrama de bosque. Pretendimos explorar la heterogeneidad analizando nuestros subgrupos prespecificados: tipo de deporte, nivel de competencia antes de la cirugía y tipo de cirugía. Sin embargo, los datos fueron insuficientes para realizar la mayoría de estos análisis. También analizamos el efecto del sesgo al comparar estudios de baja y alta calidad. Dado que la heterogeneidad fue alta para los resultados primarios, decidimos presentar los resultados de manera narrativa. ${ }^{22,23}$ El sesgo de publicación se evaluó mediante un gráfico de embudo que representa el tamaño de cada estudio en el eje X, en relación con la estimada proporción en el eje Y. Se sospecha sesgo cuando se encuentra asimetría visible en el gráfico. También realizamos la prueba de Egger para la asimetría. ${ }^{24}$

\section{RESULTADOS}

La estrategia de búsqueda arrojó 1477 registros: 1403 registros a través de la búsqueda en la base de datos y se recuperaron 58 referencias de registros de ensayos clínicos. Se excluyeron 135 referencias duplicadas, quedaron 1342 referencias únicas. Examinamos las referencias únicas leyendo títulos y resúmenes. A partir de estas referencias, identificamos 130 citas elegibles que revisamos el texto completo, de las cuales excluimos a 106. De las referencias restantes, identificamos 24 estudios que cumplieron los criterios de inclusión y tres estudios en curso. El diagrama de flujo PRISMA para la selección de estudios se resume en la Figura 1.

\section{Características del estudio y evaluación de la calidad}

Todos los estudios fueron series de casos (nivel de evidencia IV) con un tamaño medio de muestra de 56 (rango 20-200) (Tabla 1). Catorce estudios se habían llevado a cabo en Francia; cuatro, en los Estados Unidos; dos, en la Argentina; dos, en Italia y en Japón, Corea y Suiza, uno en cada país. La mediana de la edad de los participantes fue de 26 años (rango 21-31.5). Todos incluyeron predominantemente hombres con inestabilidad recurrente. Rara vez se informó el porcentaje de defecto óseo. El informe del tipo de deporte y el nivel deportivo antes de la cirugía fue variable entre los estudios, ya que dependía del sistema de clasificación utilizado por los autores. La mayoría de los participantes practicaba deportes de contacto, con una participación variable de manera "competitiva" y "recreativa". La mediana del seguimiento fue de 57 meses (rango 24-240). En 21 de los 24 estudios incluidos, se había realizado un procedimiento abierto de Latarjet; en dos, cirugías artroscópicas y, en uno, procedimientos abiertos y artroscópicos (Tabla 1). 


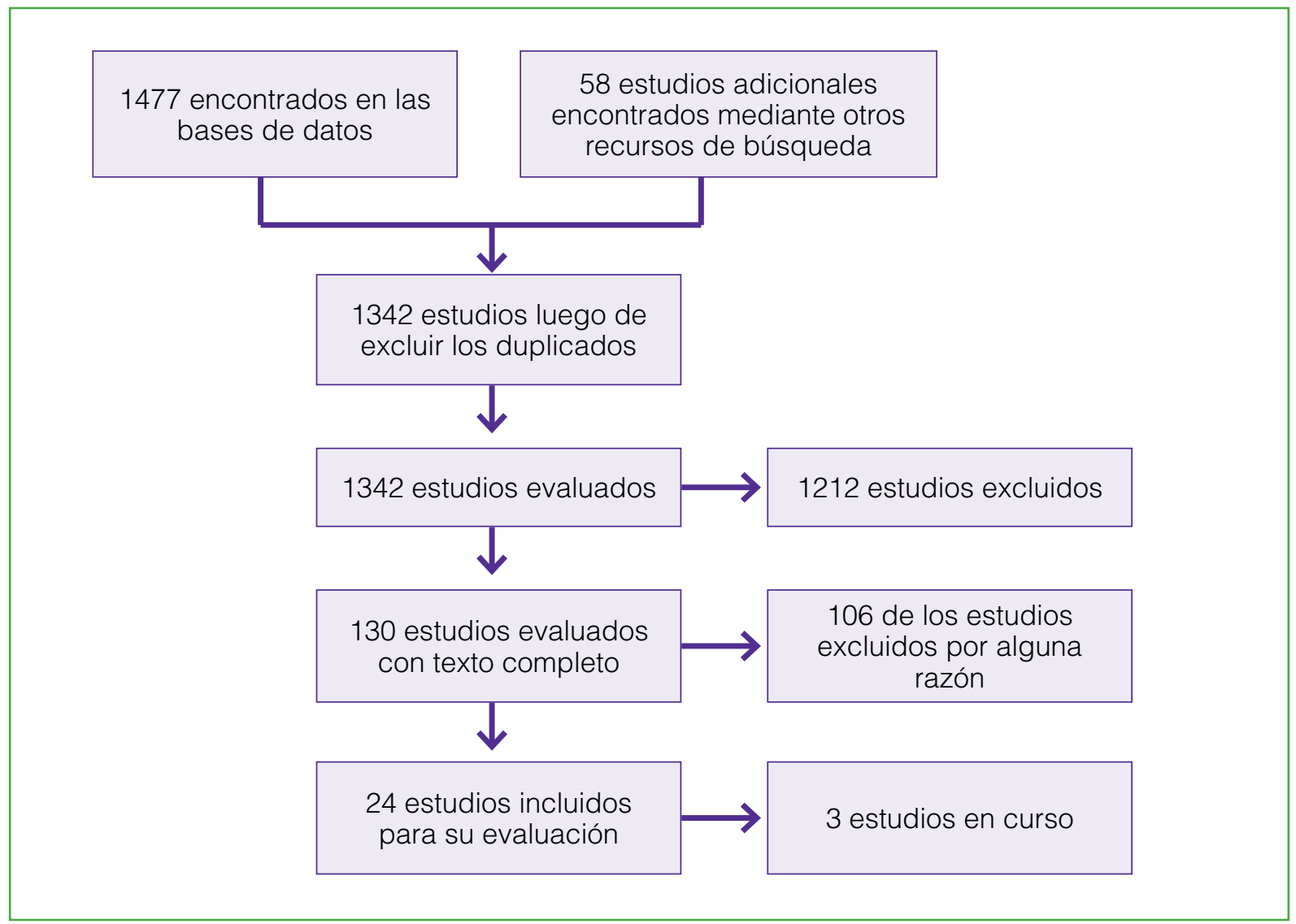

Figura 1. Método de búsqueda y selección de estudios estructurado de acuerdo con la guía PRISMA (Preferred Reporting Items for Systematic Meta-Analyses).

La mayoría de los estudios incluidos no informaron el tipo de rehabilitación, excepto un programa que describe el "programa de fortalecimiento" ${ }^{15} \mathrm{y}$ dos estudios que indican un "programa de estiramiento". ${ }^{42,47}$ Ninguno de los estudios comunicó la duración de la rehabilitación. La mayoría de los pacientes practicaba deportes de contacto y colisión $(\mathrm{n}=569)$. Solo una minoría de los estudios especificó el nombre de los deportes practicados por los pacientes incluidos. Entre estos, el más frecuente fue el rugby $(\mathrm{n}=218)$. Ningún estudio había recibido fuentes de financiación. En la Tabla 1, se detallan las características de los estudios. La mayoría de los estudios incluidos en esta revisión fueron de baja calidad (Tabla 2). En la mayoría de los estudios, faltaban características de la población y de las intervenciones. Además, en algunos, los resultados y las conclusiones estaban incompletos o no explicaban sus limitaciones. Solo tres estudios ${ }^{37,38,42}$ fueron identificados como de alta calidad. 
Tabla 1. Características de los estudios incluidos

\begin{tabular}{|c|c|c|c|c|c|c|c|c|c|c|}
\hline Autor & País & $\begin{array}{l}\text { Tamaño } \\
\text { de la } \\
\text { muestra } \\
\text { (n) }\end{array}$ & $\begin{array}{l}\text { Analizada } \\
\text { (n) }\end{array}$ & $\begin{array}{l}\text { Edad } \\
\text { prome- } \\
\text { dio }\end{array}$ & $\begin{array}{l}\text { Segui- } \\
\text { miento } \\
\text { (meses) }\end{array}$ & $\begin{array}{c}\text { Sexo } \\
\text { (\% hom- } \\
\text { bres) }\end{array}$ & $\begin{array}{c}\text { Déficit } \\
\text { óseo } \\
(\%)\end{array}$ & $\begin{array}{c}\text { Tipo } \\
\text { de } \\
\text { deporte }\end{array}$ & $\begin{array}{l}\text { Nivel previo } \\
\text { a la cirugía }\end{array}$ & $\begin{array}{l}\text { Tipo } \\
\text { de } \\
\text { cirugía }\end{array}$ \\
\hline $\begin{array}{l}\text { El Andaloussi, }{ }^{25} \\
2006\end{array}$ & Francia & 36 & 29 & 26 & 36 & 100 & NR & NR & $\begin{array}{l}\text { Principalmente } \\
\text { recreativo }\end{array}$ & Abierta \\
\hline Burkhart, ${ }^{26} 2007$ & EE.UU. & 102 & 47 & 26.5 & 59.0 & 95 & NR & NR & $\begin{array}{l}\text { Principalmente } \\
\text { competitivo }\end{array}$ & Abierta \\
\hline Neyton, ${ }^{27} 2007$ & Francia & 85 & 85 & 22.2 & 75 & 100 & 13 & Rugby & $\begin{array}{l}\text { Principalmente } \\
\text { competitivo }\end{array}$ & Abierta \\
\hline Neyton, ${ }^{28} 2012$ & Francia & 37 & 37 & 23.4 & 144 & 100 & NR & Rugby & $\begin{array}{l}\text { Principalmente } \\
\text { competitivo }\end{array}$ & Abierta \\
\hline Bessiere, ${ }^{29} 2013$ & Francia & 57 & 51 & 25 & 66 & 96 & NR & NR & $\begin{array}{l}\text { Principalmente } \\
\text { competitivo }\end{array}$ & Abierta \\
\hline Mizuno, ${ }^{30} 2013$ & Francia & 68 & 68 & 29.4 & 240 & 79 & NR & $\begin{array}{c}\text { Contacto } \\
(19 \%)\end{array}$ & $\begin{array}{l}\text { Principalmente } \\
\text { recreativo }\end{array}$ & Abierta \\
\hline Bessiere, ${ }^{31} 2014$ & Francia & 93 & 93 & 26 & 72 & 90 & NR & $\begin{array}{c}\text { Contacto } \\
(47 \%)\end{array}$ & $\begin{array}{l}\text { Principalmente } \\
\text { competitivo }\end{array}$ & Abierta \\
\hline Boileau, ${ }^{32} 2014$ & Francia & 64 & 64 & 24 & 35 & 80 & NR & $\begin{array}{l}\text { Deportes de } \\
\text { alto riesgo } \\
(84 \%)\end{array}$ & $\begin{array}{l}\text { Principalmente } \\
\text { recreativo }\end{array}$ & $\begin{array}{l}\text { Artros- } \\
\text { cópica }\end{array}$ \\
\hline Bouju, ${ }^{33} 2014$ & Francia & 78 & 78 & 26.7 & 156 & 60 & NR & $\begin{array}{c}\text { Deportes de } \\
\text { alto riesgo } \\
(59 \%)\end{array}$ & $\begin{array}{l}\text { Principalmente } \\
\text { recreativo }\end{array}$ & Abierta \\
\hline Tasaki, ${ }^{34} 2015$ & Japón & 42 & 40 & 21 & 30.5 & 99 & 12.20 & Rugby & $\begin{array}{l}\text { Principalmente } \\
\text { competitivo }\end{array}$ & $\begin{array}{l}\text { Artros- } \\
\text { cópica }\end{array}$ \\
\hline Beranger, ${ }^{35} 2016$ & Francia & 47 & 47 & 27.9 & 46.8 & 98 & NR & $\begin{array}{l}\mathrm{G} 1 / \mathrm{G} 2 \\
(64 \%)^{*}\end{array}$ & $\begin{array}{l}\text { Principalmente } \\
\text { recreativo }\end{array}$ & Abierta \\
\hline Blonna, ${ }^{36} 2016$ & Italia & 30 & 30 & 31.5 & 63 & 86 & NR & $\begin{array}{c}\text { Colisión } \\
(53 \%)\end{array}$ & NR & Abierta \\
\hline Marion, ${ }^{37} 2016$ & Francia & 58 & 43 & 27 & 29.8 & 77 & $12-25$ & NR & $\begin{array}{l}\text { Principalmente } \\
\text { recreativo }\end{array}$ & $\begin{array}{r}\text { Abierta } \\
\text { y artros- } \\
\text { cópica }\end{array}$ \\
\hline Mook, ${ }^{38} 2016$ & EE.UU. & 39 & 38 & 26 & 38 & 84 & 30 & NR & NR & Abierta \\
\hline Ropars,${ }^{39} 2016$ & Francia & 79 & 79 & 26.3 & 55 & 68 & $\begin{array}{l}\text { Solo } 36 \\
\text { pacien- } \\
\text { tes }\end{array}$ & $\begin{array}{c}14 \mathrm{G} 1,8 \mathrm{G} 2 \\
12 \mathrm{G} 3,10 \\
\mathrm{G} 3,33 \mathrm{G} 4\end{array}$ & $\begin{array}{l}\text { Principalmente } \\
\text { competitivo }\end{array}$ & Abierta \\
\hline Yang, ${ }^{40} 2016$ & EE.UU. & 52 & 42 & 23.2 & 41 & 97 & 19.70 & NR & $\begin{array}{l}\text { Principalmente } \\
\text { competitivo }\end{array}$ & Abierta \\
\hline $\begin{array}{l}\text { Zimmerman, }{ }^{41} \\
2016\end{array}$ & Suiza & 106 & 93 & 30.8 & 119 & 88 & NR & NR & NR & Abierta \\
\hline $\begin{array}{l}\text { Ranalletta, } \\
2017\end{array}$ & $\begin{array}{l}\text { Argen- } \\
\text { tina }\end{array}$ & 68 & 65 & 26.8 & 44 & 95 & 28 & $\mathrm{G} 1 / \mathrm{G} 2 * 80 \%$ & $\begin{array}{l}\text { Principalmente } \\
\text { competitivo }\end{array}$ & Abierta \\
\hline Vadalà, ${ }^{43} 2017$ & Italia & 24 & 24 & 27.2 & 24 & 91 & NR & NR & NR & Abierta \\
\hline Kee, ${ }^{44} 2017$ & Corea & 56 & 56 & 26 & 67 & 96 & NR & $\begin{array}{c}\text { Colisión } \\
(52 \%)\end{array}$ & $\begin{array}{l}\text { Principalmente } \\
\text { recreativo }\end{array}$ & Abierta \\
\hline $\begin{array}{l}\text { de l'Escalopier, }{ }^{45} \\
2018\end{array}$ & Francia & 20 & 20 & 26 & 192 & 100 & NR & NR & $\begin{array}{l}\text { Principalmente } \\
\text { competitivo }\end{array}$ & Abierta \\
\hline Baverel, ${ }^{20} 2018$ & Francia & 106 & 106 & 21 & 44 & 84 & NR & $\begin{array}{c}\text { Colisión } \\
(65 \%)\end{array}$ & $\begin{array}{l}\text { Principalmente } \\
\text { competitivo }\end{array}$ & Abierta \\
\hline Privitera, ${ }^{46} 2018$ & EE.UU. & 200 & 73 & 25.8 & 51,6 & 88 & 10.60 & $\begin{array}{l}\text { Contacto } \\
(82 \%)^{* *}\end{array}$ & $\begin{array}{l}\text { Principalmente } \\
\text { competitivo }\end{array}$ & Abierta \\
\hline $\begin{array}{l}\text { Ranalletta, }{ }^{47} \\
2018\end{array}$ & $\begin{array}{l}\text { Argen- } \\
\text { tina }\end{array}$ & 50 & 50 & 22.8 & 48 & 100 & 28 & Rugby & $\begin{array}{l}\text { Principalmente } \\
\text { competitivo }\end{array}$ & Abierta \\
\hline
\end{tabular}

$\mathrm{NR}=$ no reportado

*Según la clasificación de Allain de los deportes: de G1 a G4, según la intensidad

**Incluye: hockey sobre hielo, fútbol americano, fútbol, rugby, lacrosse, hockey sobre césped. 
Tabla 2. Evaluación de la calidad de cada estudio

\begin{tabular}{|c|c|c|c|c|c|c|c|c|}
\hline Autor/Año & Objetivo & Población & Intervención & $\begin{array}{l}\text { Segui- } \\
\text { miento }\end{array}$ & $\begin{array}{l}\text { Análisis } \\
\text { estadístico }\end{array}$ & $\begin{array}{l}\text { Resultados y } \\
\text { conclusiones }\end{array}$ & $\begin{array}{l}\text { Conflicto } \\
\text { de } \\
\text { intereses }\end{array}$ & General \\
\hline $\begin{array}{l}\text { El Andaloussi } \\
2006\end{array}$ & Bajo & Bajo & Bajo & Bajo & Bajo & Bajo & Bajo & Bajo \\
\hline Burkhart 2007 & Alto & Bajo & Alto & Bajo & Bajo & Alto & Bajo & Bajo \\
\hline Neyton 2007 & Alto & Bajo & Alto & Alto & Alto & Bajo & Bajo & Bajo \\
\hline Neyton 2012 & Alto & Bajo & Alto & Bajo & Alto & Bajo & Alto & Bajo \\
\hline Bessiere 2013 & Alto & Bajo & Bajo & Alto & Alto & Alto & Bajo & Bajo \\
\hline Mizuno 2013 & Alto & Bajo & Bajo & Bajo & Alto & Bajo & Bajo & Bajo \\
\hline Bessiere 2014 & Alto & Bajo & Bajo & Alto & Alto & Alto & Bajo & Bajo \\
\hline Boileau 2014 & Alto & Bajo & Bajo & Alto & Alto & Alto & Bajo & Bajo \\
\hline Bouju 2014 & Alto & Alto & Alto & Alto & Alto & Bajo & Alto & Bajo \\
\hline Tasaki 2015 & Alto & Bajo & Bajo & Alto & Alto & Bajo & Alto & Bajo \\
\hline $\begin{array}{l}\text { Beranger } \\
2016\end{array}$ & Alto & Bajo & Bajo & Alto & Alto & Alto & Alto & Bajo \\
\hline Blonna 2016 & Alto & Bajo & Bajo & Alto & Alto & Alto & Bajo & Bajo \\
\hline Marion 2016 & Alto & Alto & Alto & Alto & Alto & Alto & Alto & Alto \\
\hline Mook 2016 & Alto & Alto & Alto & Alto & Alto & Alto & Alto & Alto \\
\hline Ropars 2016 & Alto & Alto & Alto & Alto & Alto & Alto & Bajo & Bajo \\
\hline Yang 2016 & Alto & Bajo & Bajo & Alto & Alto & Alto & Bajo & Bajo \\
\hline $\begin{array}{l}\text { Zimmerman } \\
2016\end{array}$ & Alto & Bajo & Bajo & Alto & Alto & Bajo & Alto & Bajo \\
\hline Kee 2017 & Alto & Bajo & Bajo & Alto & Alto & Alto & Alto & Bajo \\
\hline $\begin{array}{l}\text { Ranalletta } \\
2017\end{array}$ & Alto & Alto & Alto & Alto & Alto & Alto & Alto & Alto \\
\hline Vadalà 2017 & Alto & Bajo & Bajo & Alto & Alto & Bajo & Bajo & Bajo \\
\hline $\begin{array}{l}\text { de } \\
\text { l'Escalopier } \\
2018\end{array}$ & Alto & Bajo & Bajo & Alto & Alto & Bajo & Bajo & Bajo \\
\hline Baverel 2018 & Alto & Bajo & Bajo & Alto & Alto & Alto & Bajo & Bajo \\
\hline Privitera 2018 & Alto & Bajo & Alto & Alto & Alto & Bajo & Alto & Bajo \\
\hline $\begin{array}{l}\text { Ranalletta } \\
2018\end{array}$ & Alto & Bajo & Alto & Alto & Alto & Bajo & Bajo & Bajo \\
\hline
\end{tabular}




\section{Retorno al deporte y el nivel de juego posterior}

El retorno a los deportes se evaluó en 1197 atletas en 23 estudios. No se pudo realizar un metanálisis, ya que la heterogeneidad entre los estudios fue alta (I $2=91,44 \%$ e I $2=91,73 \%$ para los resultados primarios). El rango de la tasa de retorno al deporte fue del 65\% al 100\% (Figura 2). El nivel de juego al retornar al deporte se evaluó en 19 estudios con 961 atletas. El rango de tasas de retorno a los deportes al mismo nivel de juego que antes de la cirugía fue del $23 \%$ al $100 \%$ (Figura 3). Solo siete estudios que incluyeron 355 participantes ${ }^{5,6,34,35,42,46,47}$ informaron el tiempo transcurrido para volver a practicar deportes. La mediana del tiempo de retorno al deporte fue de 6 meses, aunque el rango dentro de cada estudio varió de 1 a 36 meses. Para estos resultados, no encontramos evidencia de sesgo de publicación en los gráficos de embudo (Figura 4). La prueba de Egger no detectó asimetría ( $p>0,05)$. Diecisiete estudios informaron el nivel de competencia antes de la lesión (Figura 4). Un total que varió del 83\% al 100\% de los atletas recreativos y del 65\% al 100\% de los atletas competitivos regresó al mismo nivel de juego y esta diferencia no fue estadísticamente significativa $(\mathrm{p}=0,32)$. Exploramos las fuentes de heterogeneidad realizando análisis de subgrupos por nivel de deportes antes de la cirugía y estudios comparativos con alta y baja calidad; sin embargo, estos análisis de subgrupos no explicaron la heterogeneidad (Figuras 5 y 6). De acuerdo con la metodología GRADE, la calidad de estos resultados es muy baja, debido a las características en el diseño del estudio, sus limitaciones y la inconsistencia (Tabla 3).

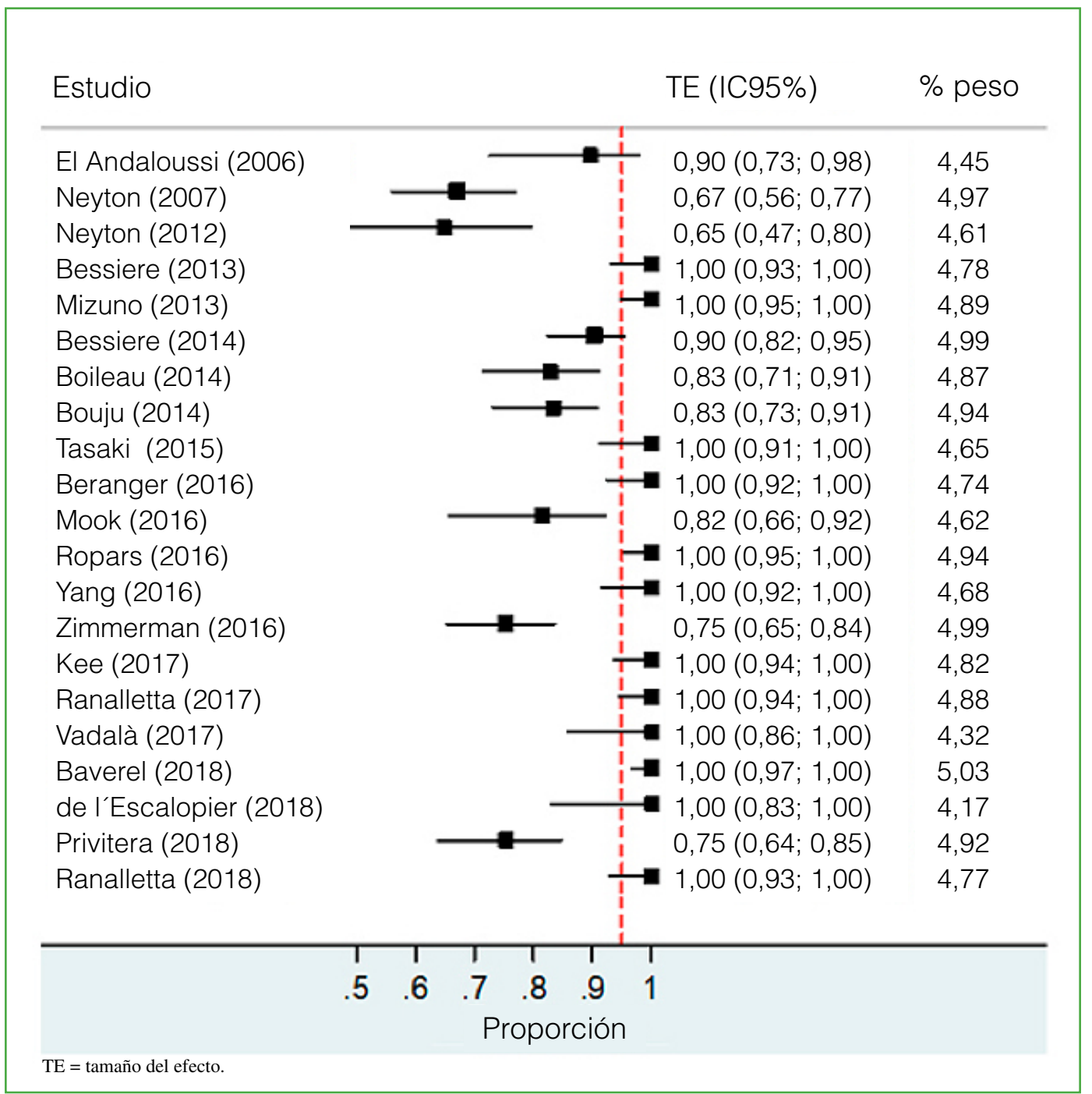

Figura 2. Retorno al deporte luego de una cirugía de Latarjet. 
Estudio

TE (IC95\%)

$\%$ peso

El Andaloussi (2006)

Neyton (2007)

Neyton (2012)

Bessiere (2013)

Mizuno (2013)

Boileau (2014)

Bouju (2014)

Tasaki (2015)

Beranger (2016)

Mook (2016)

Ropars (2016)

Yang (2016)

Kee (2017)

Ranalletta (2017)

Vadalà (2017)

Baverel (2018)

Privitera (2018)

Ranalletta (2018)

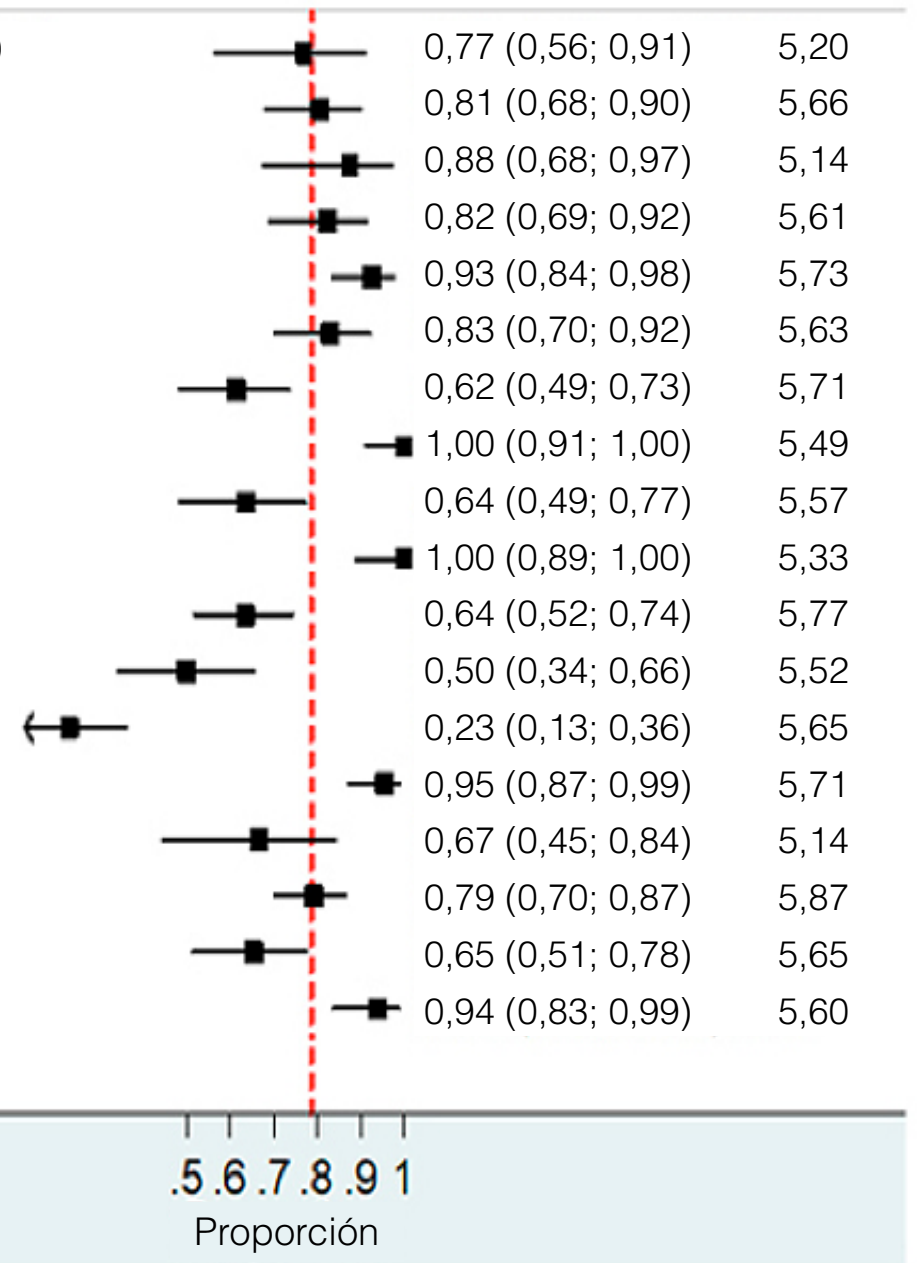

$\mathrm{TE}=$ tamaño del efecto

Figura 3. Retorno al mismo nivel deportivo luego de una cirugía de Latarjet. 


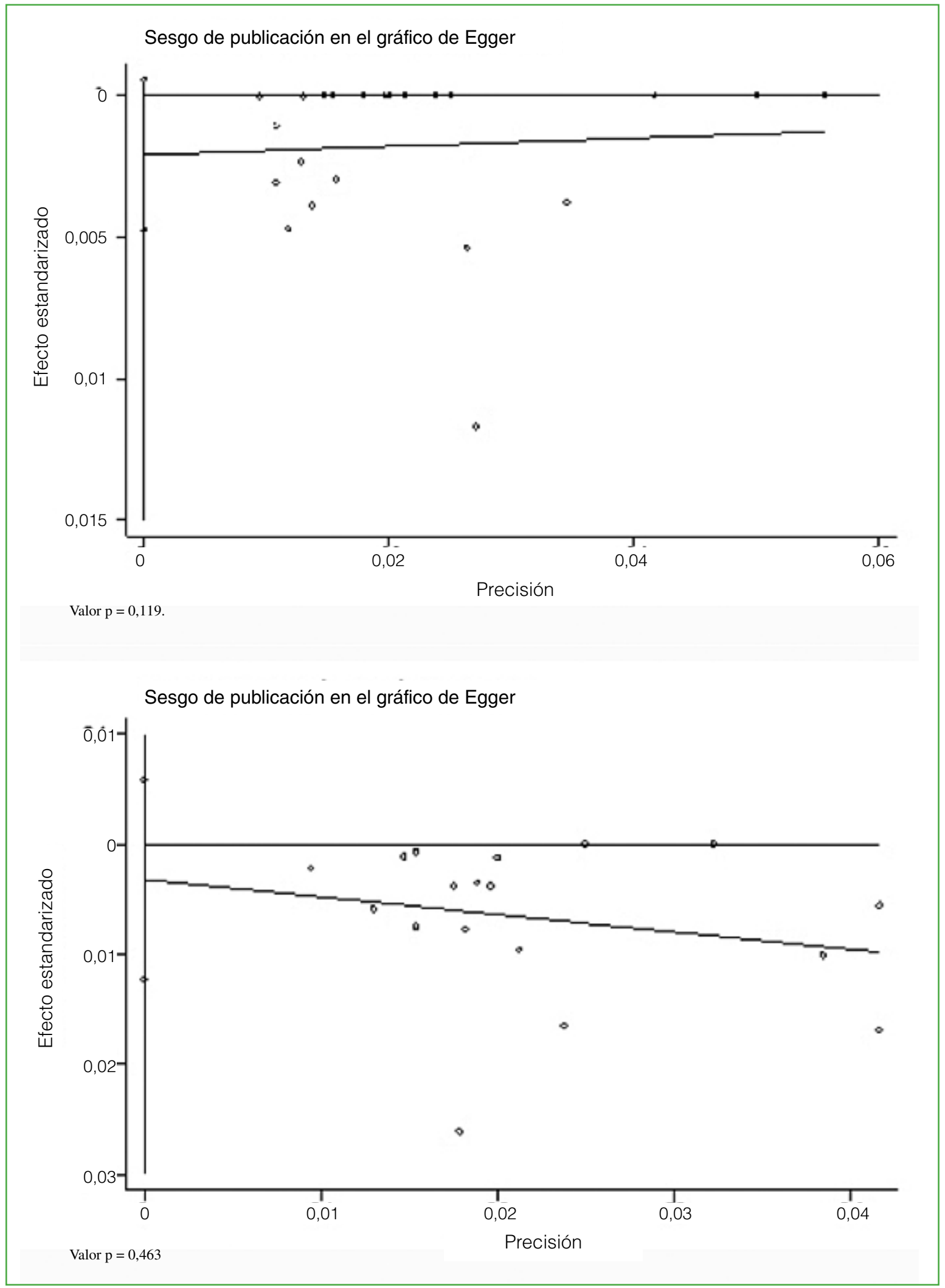

Figura 4. Gráfico de Egger y prueba de Egger para sesgo de publicación. 
Estudio

TE (IC95\%) \% peso

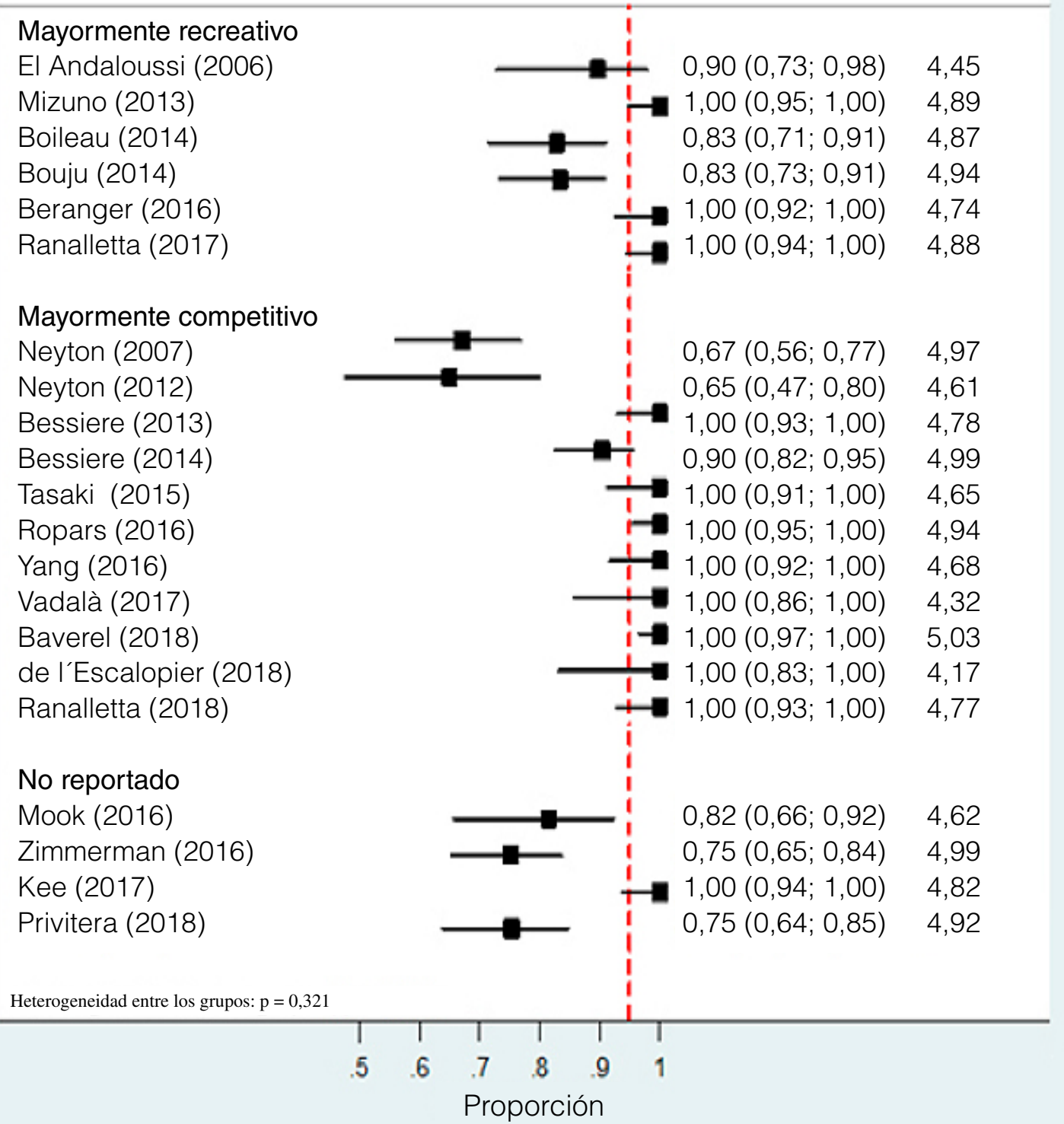

$\mathrm{TE}=$ tamaño del efecto

Figura 5. Retorno al deporte luego de la cirugía, según el nivel competitivo. 
Estudio

· TE (IC95\%)

$\%$ peso

Bajo

El Andaloussi (2006)

Neyton (2007)

Neyton (2012)

Bessiere (2013)

Mizuno (2013)

Bessiere (2014)

Boileau (2014)

Bouju (2014)

Tasaki (2015)

Beranger (2016)

Ropars (2016)

Yang (2016)

Zimmerman (2016)

Kee (2017)

Ranalletta (2017)

Baverel (2018)

de l'Escalopier

Privitera (2018)

Ranalletta (2018)

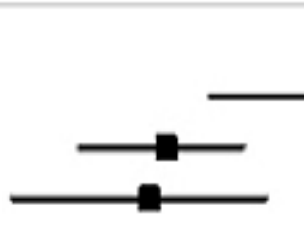

1
1
-1

$0,90(0,73 ; 0,98)$

4,45

$0,67(0,56 ; 0,77)$

4,97

$0,65(0,47 ; 0,80)$

4,61

1 .

$1,00(0,93 ; 1,00)$

4,78

-

$1,00(0,95 ; 1,00)$

4,89

$\begin{array}{rll}\longrightarrow & 0,90(0,82 ; 0,95) & 4,99 \\ & 0,83(0,71 ; 0,91) & 4,87\end{array}$

$\begin{array}{lll}\longrightarrow & 0,90(0,82 ; 0,95) & 4,99 \\ & 0,83(0,71 ; 0,91) & 4,87\end{array}$

$0,83(0,73 ; 0,91) \quad 4,94$

$\frac{1}{1} \mathbf{n} 1,00(0,91 ; 1,00) \quad 4,65$

$+1$

$1,00(0,92 ; 1,00) \quad 4,74$

-a $1,00(0,95 ; 1,00) \quad 4,94$

I- $1,00(0,92 ; 1,00) \quad 4,68$

Ranalletta (2018)

Alto

Mook (2016)

Vadalà (2017)

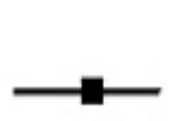

$0,75(0,65 ; 0,84)$

4,99

$1,00(0,94 ; 1,00) \quad 4,82$

$1,00(0,94 ; 1,00)$

4,88

$1,00(0,97 ; 1,00)$

5,03

$1,00(0,83 ; 1,00)$

4,17

$0,75(0,64 ; 0,85)$

4,92

$1,00(0,93 ; 1,00)$

4,77
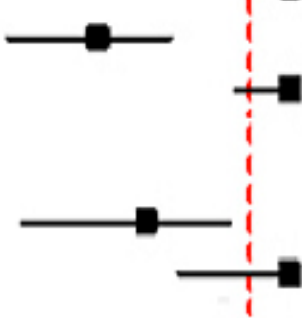

$0,82(0,66 ; 0,92)$

4,62

$1,00(0,86 ; 1,00)$

4,32

Heterogeneidad entre grupos: $\mathrm{p}=0,373$

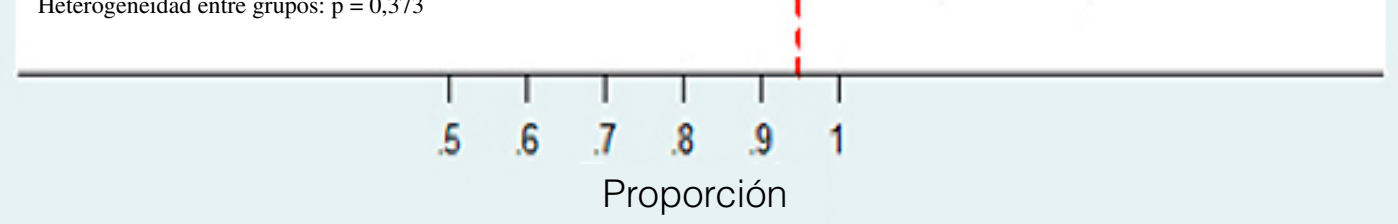

$\mathrm{TE}=$ tamaño del efecto

Figura 6. Retorno al deporte después de la cirugía. Subgrupo estudiado, según la calidad de los estudios. 
Tabla 3. Resumen de los principales resultados de esta revisión utilizando criterios GRADE

Paciente o población: Deportistas con inestabilidad de hombro

Ajustes: Pacientes internados - Seguimiento ambulatorio; estudios de Francia (14), EE.UU. (4), Argentina (2), Italia (2), Japón (1), Corea (1), Suiza (1)

Cirugía: Cirugía de Latarjet abierta o artroscópica

\section{Ítems evaluados \\ Retorno al deporte \\ Cantidad de pacientes que \\ volvieron al deporte luego de la \\ cirugía}

Retorno al mismo nivel

deportivo

Cantidad de pacientes que

retornaron al mismo nivel

deportivo*

\section{Pacientes}

estudiados

1197

(23 estudios observacionales)

961

(19 estudios observacionales)

Certeza de la evidencia
(GRADE)

$\oplus \bigcirc \bigcirc \bigcirc$

Muy bajo ${ }^{\mathrm{a}, \mathrm{b}}$

$\oplus \bigcirc \bigcirc \bigcirc$

Muy bajo ${ }^{\mathrm{a}, \mathrm{b}}$
Rango de proporción de los pacientes

65-100 cada 100 pacientes

"Dentro de los pacientes que retornaron al deporte; CI: Intervalo de confianza.

\section{GRADE Grados de evidencia del grupo estudiado}

Alta certeza: Estamos muy confiados de que el verdadero efecto se acerca a la estimacion real del efecto.

Moderada certeza: Estamos moderadamente confiados en el efecto estimado: el verdadero efecto está cerca del efecto esperado, pero existen posibilidades de que sea sustancialmetes diferente.

Baja certeza: Creemos que el efecto encontrado es limitado: el verdadero efecto puede ser sustancialemte diferente del encontrado.

Muy baja certeza: Tenemos muy poca confianza en el efecto estimado: el verdadero efecto es probablemente diferente del efecto encontrado.

\section{Explicaciones}

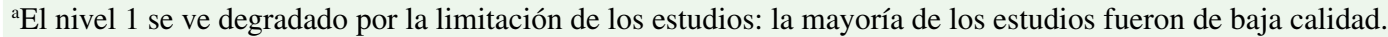

${ }^{b}$ El nivel 1 se ve degradado por la inconsistencia: alta heterogeneidad estadística de los estudios (>90\%).

\section{Complicaciones y revisiones posoperatorias}

Diecisiete estudios informaron complicaciones posoperatorias en 1158 participantes. El rango de la tasa de complicaciones fue del $2 \%$ al $31 \%$ (Figura 7). Las principales complicaciones fueron problemas de injerto (falta de consolidación, fragmentación), problemas del tornillo (rotura/prominencia intrarticular) e infecciones. Once estudios informaron revisiones en 764 pacientes. ${ }^{20,27,28,30-32,38,40-42,46}$ Pocos estudios informaron la cantidad de revisiones, pero las más comunes fueron por complicaciones de los tornillos $(n=8 \text { de } 486)^{27,30,40,41}$ y recurrencia de la inestabilidad $(\mathrm{n}=4$ de 311$) .20,28,31,42,46$

\section{DISCUSIÓN}

Los principales hallazgos de este estudio fueron que, con una evidencia muy baja, la mayoría de los atletas con inestabilidad glenohumeral anterior recurrente regresaron al deporte; sin embargo, el nivel mantenido después de la estabilización del hombro con el procedimiento de Latarjet varió sustancialmente en un seguimiento medio de 57 meses. Además, el tiempo promedio para regresar a los deportes fue de 6 meses y los resultados parecieron ser igualmente favorables tanto en atletas competitivos como recreativos.

En nuestro estudio, el 65-100\% de los pacientes pudo regresar a practicar deportes, y el 23-100\% regresó al mismo nivel que antes de la lesión. Curiosamente, no se observaron diferencias significativas con respecto al retorno al deporte y el nivel alcanzado por los atletas cuando comparamos atletas competitivos y recreativos. Pocos autores han analizado los resultados del procedimiento de Latarjet en función de las actividades deportivas de los pacientes. Baverel y cols. ${ }^{20}$ compararon retrospectivamente 106 pacientes divididos en dos grupos según las actividades 


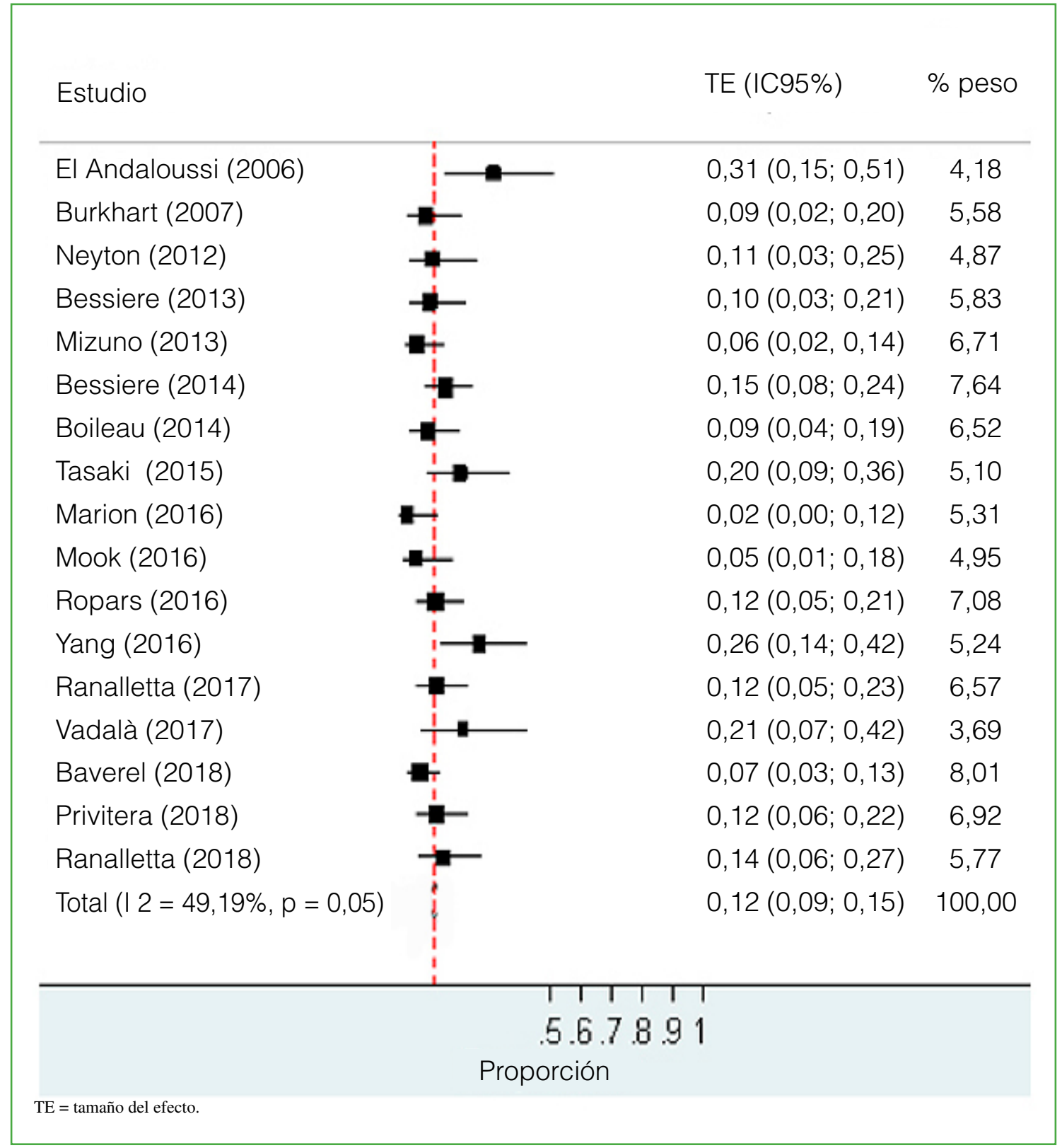

Figura 7. Complicaciones luego de una cirugía de Latarjet.

deportivas: 57 (54\%) atletas competitivos y 49 (46\%) atletas recreativos. Estos autores hallaron que el 100\% de los atletas competitivos y el $69 \%$ de los atletas recreativos reanudaron su práctica deportiva. Además, el $79 \%$ de los atletas competitivos y el $43 \%$ de los atletas recreativos alcanzaron el mismo nivel que antes de su lesión. Plantean la hipótesis de que la diferencia podría deberse a que los atletas competitivos son más disciplinados con sus programas de rehabilitación o tienen una mejor propiocepción y fuerza muscular en su hombro. Por otro lado, Beranger y cols. ${ }^{35}$ informaron que el regreso al deporte después de la estabilización del hombro con un procedimiento de Latarjet fue posible en todos los atletas competitivos y recreativos después de 6.3 meses y el 78,7\% de los pacientes retomó su nivel anterior a la lesión. Otros autores también informaron excelentes resultados funcionales y altas tasas de retorno al deporte en atletas recreativos..$^{35,42,47}$ 
El tiempo medio de retorno al deporte fue de 6 meses, aunque el rango dentro de cada estudio varió de 1 a 36 meses. Esto muestra la falta de consenso entre los autores con respecto a los criterios utilizados para permitir que los atletas regresen a la competencia. En una revisión sistemática reciente, Ciccoti y cols. ${ }^{48}$ evaluaron los criterios de retorno al deporte luego de una estabilización quirúrgica para la inestabilidad anterior traumática del hombro. Identificaron 13 posibles criterios para el retorno al deporte sin llegar a un consenso en ninguno de ellos. Además, observaron una variabilidad significativa entre los autores que utilizan los mismos criterios. Como ejemplo, el "tiempo" fue el criterio más utilizado para el regreso al juego; sin embargo, los puntos de tiempo específicos para el retorno al deporte variaron de 1.5 meses a 12 meses entre los distintos estudios. Del mismo modo, es una limitación significativa de la bibliografía disponible que los protocolos posoperatorios no se informan o se describen brevemente sin la metodología necesaria para ser reproducibles. En nuestra revisión sistemática, ningún estudio describió un protocolo completo de rehabilitación posoperatoria y solo cinco $^{20,30,33,42,47}$ especificaron los criterios para el retorno al deporte, aparte de un punto fijo en el tiempo. No está claro el momento en que los pacientes pueden volver a jugar sin restricciones después del procedimiento de Latarjet y sería muy útil crear una lista de verificación integral de regreso al juego basada en la evidencia para la estabilización anterior del hombro.

Algunos autores evaluaron la relación entre las tasas de retorno al deporte y el tipo de deporte. La mayoría de los estudios mostró que la tasa de retorno al deporte era alta, incluso en los deportes más riesgosos. Privitera y cols. ${ }^{46}$ y Ranalletta y cols. ${ }^{47}$ publicaron las dos series más grandes que evalúan los resultados de los atletas de contacto o colisión tras el procedimiento de Latarjet. La tasa de retorno al deporte fue del $75 \%$ y $100 \%$, respectivamente. Otros autores también informaron altas tasas de retorno al deporte en atletas de colisión, que oscilan entre el 65\% y el $97 \% .27,28,35,42 \mathrm{El}$ regreso al deporte podría verse comprometido en los atletas con movimientos por sobre la cabeza. Berenger y cols. ${ }^{35}$ informaron que los pacientes que practicaban deportes con movimientos por sobre la cabeza tenían más probabilidades de jugar a un nivel más bajo o de cambiar de deporte después de la cirugía.

Solo unos pocos estudios analizaron el regreso al deporte después del procedimiento de Latarjet como revisión de estabilizaciones fallidas en atletas. Ranalletta y cols. ${ }^{42}$ evaluaron a 68 atletas tratados con el procedimiento de Latarjet para la inestabilidad recurrente del hombro después de una cirugía de estabilización previa fallida. Los autores no encontraron diferencias significativas con respecto al regreso al deporte y al nivel alcanzado por los atletas cuando compararon los procedimientos primarios y de revisión. Sin embargo, la mayoría de los pacientes en el grupo de revisión tenía solo un procedimiento previo (media 1,26 procedimientos). Privitera y cols. ${ }^{46}$ informaron los resultados del procedimiento de Latarjet para la inestabilidad glenohumeral anterior recurrente en 73 atletas. $\mathrm{Al}$ igual que los autores anteriores, hallaron que, en un seguimiento promedio de 52 meses, la tasa de retorno al deporte fue similar cuando el procedimiento de Latarjet se realizó como un procedimiento de estabilización primaria $(72 \%)$ y cuando se realizó para pacientes con solo un procedimiento de estabilización previo (75\%). Sin embargo, para los atletas con más de dos procedimientos de estabilización previos, la tasa de retorno al deporte fue significativamente menor (39\%).

Las complicaciones asociadas con el procedimiento de Latarjet, especialmente en pacientes jóvenes activos, son un punto por considerar. Las que se comunican con más frecuencia son: implantes flojos, fractura o falta de consolidación del injerto coracoideo, recurrencia de la inestabilidad, infección, hombro congelado, formación de hematomas, complicaciones neurológicas y artritis. ${ }^{49,50}$ Una revisión reciente informó una tasa general de complicaciones del $30 \% .^{50}$ Nuestro estudio halló una tasa de complicaciones que varió entre el $2 \%$ y el $31 \%$. Específicamente con respecto a las recurrencias, el procedimiento de Latarjet parece suficiente para lograr la estabilidad necesaria en pacientes con inestabilidad glenohumeral recurrente incluso en pacientes de alta demanda, como atletas de contacto y colisión. Paulino Oereira y cols., ${ }^{51}$ en una revisión sistemática reciente que evaluó a 802 pacientes en 11 estudios, observaron una tasa de recurrencia general del 2,7\% sin diferencias significativas entre los deportes de colisión y los que no lo son.

\section{LIMITACIONES}

Este estudio tiene varias limitaciones. Primero, como con cualquier revisión sistemática, hay estudios que podrían haberse perdido con nuestros criterios de búsqueda y los sesgos inherentes de cada estudio incluido pueden haber influido en nuestros resultados. Sin embargo, realizamos una búsqueda exhaustiva en múltiples bases de datos siguiendo un protocolo prespecificado para una revisión sistemática, presentando una evaluación detallada del riesgo de sesgo y destacando específicamente las limitaciones importantes de cada estudio para disminuir el riesgo de mala interpretación de los datos. En segundo lugar, la mayoría de los estudios incluidos en el análisis fueron series de casos retrospectivos, aplicándose a estos todas las limitaciones de los estudios retrospectivos basados en 
series de casos. En tercer lugar, los detalles de las técnicas quirúrgicas y los protocolos de estudios por imágenes posoperatorios no se estandarizaron en todos los estudios, lo que también podría constituir un factor de confusión. Por ejemplo, algunos autores efectuaron una reparación capsulolabral con arpones, ${ }^{32,34}$ otros solo suturaron remanente del ligamento coracoacromial a la cápsula anterior ${ }^{28,38,46}$ y otros no repararon el complejo capsulolabral. ${ }^{42,47}$

\section{CONCLUSIONES}

La evidencia de muy baja calidad indica que un alto porcentaje de los atletas con inestabilidad glenohumeral anterior recurrente regresaron al deporte; sin embargo, el nivel mantenido después de la estabilización del hombro con el procedimiento de Latarjet varió sustancialmente. El tiempo promedio para regresar al deporte fue de 6 meses y los resultados fueron igualmente satisfactorios en atletas competitivos y recreativos.

Conflicto de intereses: Los autores no declaran conflictos de intereses.

ORCID de L. Rossi: https://orcid.org/0000-0002-1397-2402

ORCID de J. V. A. Franco: https://orcid.org/0000-0002-2903-6870

ORCID de C. M. Escobar Liquitay: https://orcid.org/0000-0003-0411-899X
ORCID de I. Pasqualini: https://orcid.org/0000-0002-9551-827X ORCID de M. Ranalletta: https://orcid.org/0000-0002-9145-4010

\section{BIBLIOGRAFÍA}

1. Gowd AK, Liu JN, Cabarcas BC, Garcia GH, Cvetanovich GL, Provencher MT, Verma NN. Management of recurrent anterior shoulder instability with bipolar bone loss: a systematic review to assess critical bone loss amounts. Am J Sports Med 2018;27:363546518791555. https://doi.org/10.1177/0363546518791555

2. Willemot LB, Elhassan BT, Verborgt O. Bony reconstruction of the anterior glenoid rim. J Am Acad Orthop Surg 2018;26(10):e207-e218. https://doi.org/10.5435/JAAOS-D-16-00649

3. Degen RM, Camp CL, Werner BC, Dines DM, Dines JS. Trends in bone-block augmentation among recently trained orthopaedic surgeons treating anterior shoulder instability. J Bone Joint Surg Am 2016;98(13):e56. https://doi.org/10.2106/JBJS.15.01478

4. Rabinowitz J, Friedman R, Eichinger JK. Management of glenoid bone loss with anterior shoulder instability: indications and outcomes. Curr Rev Musculoskelet Med 2017;10(4):452-62. https://doi.org/10.1007/s12178-017-9439-y

5. Riff AJ, Frank RM, Sumner S, Friel N, Bach BR Jr, Verma NN, Romeo AA. Trends in shoulder stabilization techniques used in the United States based on a large private-payer database. Orthop J Sports Med 2017;5(12):2325967117745511. https://doi.org/10.1177/2325967117745511

6. Ekhtiari S, Horner NS, Bedi A, Ayeni OR, Khan M. The learning curve for the Latarjet procedure: a systematic review. Orthop J Sports Med 2018;6(7):2325967118786930. https://doi.org/10.1177/2325967118786930

7. Dines JS, Dodson CC, McGarry MH, Oh JH, Altchek DW, Lee TQ. Contribution of osseous and muscular stabilizing effects with the Latarjet procedure for anterior instability without glenoid bone loss. $J$ Shoulder Elbow Surg 2013;22(12):1689-94. https://doi.org/10.1016/j.jse.2013.02.014

8. Yamamoto N, Muraki T, An KN, Sperling JW, Cofield RH, Itoi E, et al. The stabilizing mechanism of the Latarjet procedure: a cadaveric study. J Bone Joint Surg Am 2013;95(15):1390-7. https://doi.org/10.2106/JBJS.L.00777

9. An VV, Sivakumar BS, Phan K, Trantalis J. A systematic review and meta-analysis of clinical and patient-reported outcomes following two procedures for recurrent traumatic anterior instability of the shoulder: Latarjet procedure vs. Bankart repair. J Shoulder Elbow Surg 2016;25(5):853-63. https://doi.org/10.1016/j.jse.2015.11.001

10. Lädermann A, Böhm E, Tay E, Scheibel M. Bone-mediated anteroinferior glenohumeral instability : Current concepts. Orthopade 2018;47(2):129-38. https://doi.org/10.1007/s00132-017-3511-6

11. Porter DA, Birns M, Hobart SJ, Kowalsky M, Galano GJ. Arthroscopic treatment of osseous instability of the shoulder. HSS J 2017;13(3):292-301. https://doi.org/10.1007/s11420-017-9553-9 
12. Tjong VK, Devitt BM, Murnaghan ML, Ogilvie-Harris DJ, Theodoropoulos JS. A qualitative investigation of return to sport after arthroscopic Bankart repair: beyond stability. Am J Sports Med 2015;43(8):2005-11. https://doi.org/10.1177/0363546515590222

13. Higgins JPT, Lasserson T, Chandler J, Tovey D, Churchill R. Methodological Expectations of Cochrane Intervention [Internet]. London: Cochrane; Version July 2019. Standards for the conduct of new Cochrane Intervention (C1-75). [cited 2019 sept 03]. Disponible en:

https://community.cochrane.org/mecir-manual/standards-conduct-new-cochrane-intervention-reviews-c1-75

14. Higgins JPT, Green S. Cochrane Handbook for Systematic Reviews of Interventions Version 5.1.0 [updated March 2011]. The Cochrane Collaboration, 2011. Disponible en: www.handbook.cochrane.org

15. Moher D, Liberati A, Tetzlaff J, Altman DG, Group P. Preferred reporting items for systematic reviews and metaanalyses: the PRISMA statement. Int J Surg 2010;8:336-41. https://doi.org/10.1016/j.ijsu.2010.02.007

16. Moga C, Guo B, Schopflocher D, Harstall C. Development of a quality appraisal tool for case series studies using a modified Delphi technique [Internet]. Edmonton AB: Institute of Health Economics; 2012:71 [cited 2019 sept 03]. Disponible en:

https://www.ihe.ca/advanced-search/development-of-a-quality-appraisal-tool-for-case-series-studies-using-a-modified-delphi-technique

17. Schünemann H, Brożek J, Guyatt G, Oxman A (eds). Handbook for grading the quality of evidence and the strength of recommendations using the GRADE approach. Updated October 2013 [Internet]. The GRADE Working Group; 2013 [cited 2019 sept 03]. Disponible en: https://gdt.gradepro.org/app/handbook/handbook.html\#h.hnedbo8gqjqk

18. El Andaloussi Y, Arssi M, Zaouari T, Benhima MA, Cohen D, Trafeh M, et al. Instabilité antérieure de l'épaule chez les sportifs À propos de 36 cas. J Traumatol Sport 2006;23:148-52. https://doi.org/10.1016/S0762-915X(06)71431-0

19. Nyaga VN, Arbyn M, Aerts M. Metaprop: a Stata command to perform meta-analysis of binomial data. Arch Public Health 2014;72(1):39. https://doi.org/10.1186/2049-3258-72-39

20. Baverel L, Colle PE, Saffarini M, Anthony Odri G, Barth J. Open Latarjet procedures produce better outcomes in competitive athletes compared with recreational athletes: a clinical comparative study of 106 athletes aged under 30 years. Am J Sports Med 2018;46(6):1408-15. https://doi.org/10.1177/0363546518759730

21. Higgins JPT, Green S (eds). Cochrane Handbook for Systematic Reviews of Interventions Version 5.1.0 [updated March 2011][Internet]. The Cochrane Collaboration; 2011. Chapter 9, Analysing data and undertaking metaanalyses. [cited 2019 sept 03]. Disponible en: www.handbook.cochrane.org

22. Cote MP, Lubowitz JH, Rossi MJ, Brand JC. Reviews pooling heterogeneous, low-evidence, high-bias data result in incorrect conclusions: but heterogeneity is an opportunity to explore. Arthroscopy 2018;34(12):3126-8. https://doi.org/10.1016/j.arthro.2018.10.005

23. Harris JD, Brand JC, Cote MP, Faucett SC, Dhawan A. Research pearls: the significance of statistics and perils of pooling. Part 1: Clinical versus statistical significance. Arthroscopy 2017;33(6):1102-12. https://doi.org/10.1016/j.arthro.2017.01.053

24. Hunter JP, Saratzis A, Sutton AJ, Boucher RH, Sayers RD, Bown MJ. In meta-analyses of proportion studies, funnel plots were found to be an inaccurate method of assessing publication bias. J Clin Epidemiol 2014;67(8):897-903. https://doi.org/10.1016/j.jclinepi.2014.03.003

25. GRADEpro GDT [Software]. McMaster University and Evidence Prime; c2015 [cited 2019 Sep 03]. Disponible en: gradepro.org

26. Burkhart SS, De Beer JF, Barth JR, Cresswell T, Roberts C, Richards DP. Results of modified Latarjet reconstruction in patients with anteroinferior instability and significant bone loss. Arthroscopy 2007;23:1033-41. https://doi.org/10.1016/j.arthro.2007.08.009

27. Neyton L, Dagher E, Jouve F, Josserand LN, Walch G. Chronic anterior glenohumeral instability in rugby player. Series of 85 Latarjet procedures with medium follow-up of seven years. J Traumatol Sport 2007;24(3):122-7. https://doi.org/10.1016/j.jts.2007.06.007

28. Neyton L, Young A, Dawidziak B, Visona E, Hager J-P, Fournier Y, et al. Surgical treatment of anterior instability in Rugby Union players: clinical and radiographic results of the Latarjet-Patte procedure with minimum 5-year followup. J Shoulder Elbow Surg 2012;21:1721-7. https://doi.org/10.1016/j.jse.2012.01.023

29. Bessiere C, Trojani C, Pélégri C, Carles M, Boileau P. Coracoid bone block versus arthroscopic Bankart repair: a comparative paired study with 5-year follow-up. Orthop Traumatol Surg Res 2013;99(2):123-30. https://doi.org/10.1016/j.otsr.2012.12.010

30. Mizuno N, Denard PJ, Raiss P, Melis B, Walch G. Long-term results of the Latarjet procedure for anterior instability of the shoulder. J Shoulder Elbow Surg 2014;23(11):1691-9. https://doi.org/10.1016/j.jse.2014.02.015 
31. Bessiere C, Trojani C, Carles M, Mehta SS, Boileau P. The open Latarjet procedure is more reliable in terms of shoulder stability than arthroscopic Bankart repair. Clin Orthop Relat Res 2014;472(8):2345-51. https://doi.org/10.1007/s11999-014-3550-9

32. Boileau P, Thélu CÉ, Mercier N, Ohl X, Houghton-Clemmey R, Carles M, et al. Arthroscopic Bristow-Latarjet combined with Bankart repair restores shoulder stability in patients with glenoid bone loss. Clin Orthop Relat Res 2014;472(8):2413-24. https://doi.org/10.1007/s11999-014-3691-x

33. Bouju Y, Gadéa F, Stanovici J, Moubarak H, Favard L. Shoulder stabilization by modified Latarjet-Patte procedure: results at a minimum 10 years' follow-up, and role in the prevention of osteoarthritis. Orthop Traumatol Surg Res 2014;100(4 Suppl): S213-8. https://doi.org/10.1016/j.otsr.2014.03.010

34. Tasaki A, Morita W, Yamakawa A, Nozaki T, Kuroda E, Hoshikawa Y, et al. Combined arthroscopic Bankart repair and coracoid process transfer to anterior glenoid for shoulder dislocation in rugby players: evaluation based on ability to perform sport-specific movements effectively. Arthroscopy 2015;31(9):1693-701. https://doi.org/10.1016/j.arthro.2015.03.013

35. Beranger JS, Klouche S, Bauer T, Demoures T, Hardy P. Anterior shoulder stabilization by Bristow-Latarjet procedure in athletes: return-to-sport and functional outcomes at minimum 2-year follow-up. Eur J Orthop Surg Traumatol 2016;26:277-82. https://doi.org/10.1007/s00590-016-1751-5

36. Blonna D, Bellato E, Caranzano F, Assom M, Rossi R, Castoldi F. Arthroscopic Bankart repair versus open BristowLatarjet for shoulder instability: a matched-pair multicenter study focused on return to sport. Am J Sports Med 2016;44(12):3198-3205. https://doi.org/10.1177/0363546516658037

37. Marion B, Klouche S, Deranlot J, Bauer T, Nourissat G, Hardy P. A prospective comparative study of arthroscopic versus mini-open Latarjet procedure with a minimum 2-year follow-up. Arthroscopy 2017;33(2):269-77. https://doi.org/10.1016/j.arthro.2016.06.046

38. Mook WR, Petri M, Greenspoon JA, Horan MP, Dornan GJ, Millett PJ. Clinical and anatomic predictors of outcomes after the Latarjet procedure for the treatment of anterior glenohumeral instability with combined glenoid and humeral bone defects. Am J Sports Med 2016;44(6):1407-16. https://doi.org/10.1177/0363546516634089

39. Ropars M, Cretual A, Kaila R, Bonan I, Hervé A, Thomazeau H. Diagnosis and treatment of anteroinferior capsular redundancy associated with anterior shoulder instability using an open Latarjet procedure and capsulorrhaphy. Knee Surg Sports Traumatol Arthrosc 2016;24(12):3756-64. https://doi.org/10.1007/s00167-015-3621-9

40. Yang JS, Mazzocca AD, Cote MP, Edgar CM, Arciero RA. Recurrent anterior shoulder instability with combined bone loss: treatment and results with the modified Latarjet procedure. Am J Sports Med 2016;44(4):922-32. https://doi.org/10.1177/0363546515623929

41. Zimmermann SM, Scheyerer MJ, Farshad M, Catanzaro S, Rahm S, Gerber C. Long-term restoration of anterior shoulder stability: a retrospective analysis of arthroscopic Bankart repair versus open Latarjet procedure. J Bone Joint Surg Am 2016;98(23):1954-61. https://doi.org/10.2106/JBJS.15.01398

42. Ranalletta M, Rossi LA, Bertona A, Tanoira I, Maignon GD, Bongiovanni SL. Modified Latarjet procedure without capsulolabral repair for the treatment of failed previous operative stabilizations in athletes. Arthroscopy 2018;34(5):1421-7. https://doi.org/10.1016/j.arthro.2017.12.006

43. Vadalà A, Lanzetti RM, De Carli A, Lupariello D, Guzzini M, Desideri D, et al. Latarjet procedure: evolution of the bone block and correspondent clinical relevance-a clinical and radiological study. Musculoskelet Surg 2017;101(Suppl 2):113-20. https://doi.org/10.1007/s12306-017-0482-Z

44. Kee YM, Kim JY, Kim HJ, Lim CT, Rhee YG. Return to sports after the Latarjet procedure: high return level of noncollision athletes. Knee Surg Sports Traumatol Arthrosc 2018;26(3):919-25. https://doi.org/10.1007/s00167-017-4775-4

45. de l'Escalopier N, Barbier O, Demoures T, Ollat D, Versier G. Long-term results of a monocentric series of soldiers after Latarjet procedure for anterior shoulder instability. Implications for the assessment of soldiers' medical ability. Mil Med 2018;183(1-2):e134-e137. https://doi.org/10.1093/milmed/usx040

46. Privitera DM, Sinz NJ, Miller LR, Siegel EJ, Solberg MJ, Daniels SD, et al. Clinical outcomes following the Latarjet procedure in contact and collision athletes. J Bone Joint Surg Am 2018;100(6):459-65. https://doi.org/10.2106/JBJS.17.00566

47. Ranalletta M, Rossi LA, Bertona A, Tanoira I, Alonso Hidalgo I, Maignon GD, et al. Modified Latarjet without capsulolabral repair in rugby players with recurrent anterior glenohumeral instability and significant glenoid bone loss. Am J Sports Med 2018;46(4):795-800. https://doi.org/10.1177/0363546517749586 
48. Ciccotti MC, Syed U, Hoffman R, Abboud JA, Ciccotti MG, Freedman KB. Return to play criteria following surgical stabilization for traumatic anterior shoulder instability: a systematic review. Arthroscopy 2018;34(3):90313. https://doi.org/10.1016/j.arthro.2017.08.293

49. Domos P, Lunini E, Walch G. Contraindications and complications of the Latarjet procedure. Shoulder Elbow 2018;10(1):15-24. https://doi.org/10.1177/1758573217728716

50. Griesser MJ, Harris JD, McCoy BW, Hussain WM, Jones MH, Bishop JY, et al. Complications and re-operations after Bristow-Latarjet shoulder stabilization: a systematic review. J Shoulder Elbow Surg 2013;22:286-292. https://doi.org/10.1016/j.jse.2012.09.009

51. Paulino Pereira NR, van der Linde JA2, Alkaduhimi H2, Longo UG3, van den Bekerom MPJ. Are collision athletes at a higher risk of re-dislocation after an open Bristow-Latarjet procedure? A systematic review and meta-analysis. Shoulder Elbow 2018;10(2):75-86. https://doi.org/10.1177/1758573217728290 